\title{
EQUAL-GAIN COMBINING WITH INTERFERENCE MITIGATION FOR MOLECULAR TYPE HOPPING ASSISTED MOLECULAR SHIFT KEYING SYSTEMS
}

\author{
Weidong Gao, Terrence Mak and Lie-Liang Yang ${ }^{1}$ \\ ${ }^{1}$ School of Electronics and Computer Science, University of Southampton, S017 1BJ, UK.
}

NOTE: Corresponding author: Lie-Liang Yang, lly@ecs.soton.ac.uk

\begin{abstract}
In multiple access molecular diffusive communications, many nano-machines exchange information and fuse data through a common Diffusive Molecular Communication (DMC) channel. Hence, there is Multiple-Access Interference (MAI), which should be sufficiently mitigated so as to achieve reliable communications. In this paper, we propose a novel lowcomplexity detection scheme, namely Equal-Gain Combining with Interference Mitigation (EGC-IM), for signal detection in the Molecular Type Hopping assisted Molecular Shift Keying (MTH-MoSK) DMC systems. By removing a number of entries from each row of the detection matrix formed during detection, the EGC-IM scheme shows its potential to significantly mitigate MAI and hence, outperform the conventional EGC scheme. Furthermore, the EGC-IM scheme has lower complexity than the conventional EGC scheme and therefore, it is beneficial for practical implementation.
\end{abstract}

Keywords - Diffusive molecular communications, equal-gain combining, interference mitigation, molecular shift keying, multiple-access

\section{INTRODUCTION}

Because of the manufacturing and size limitations, individual nano-machines are only capable of finishing simple missions. To enhance the capability of nanomachines, nano-networking built on the basis of individual nano-machines via information exchange and cooperation is required to complete relatively complex tasks. Molecular Communications (MC) has been recognized to be a promising solution to implement communication between nano-devices, and has received a lot of research interest in recent years [1]. In nano-networking, multiple-access communications constitute an important technique, which considers information transmission between multiple transmitter nano-machines and one common receiver $[2,3,4,5,6]$.

According to different information particles and the mechanisms of propagation, various types of multipleaccess techniques have been proposed/introduced to MC in literature. For instance, in [2, 3], the authors introduced Molecular Code-Division Multiple-Access (MCDMA), which assigns different signature codes to different nano-machines, for supporting them to transmit information simultaneously with a common access point. At the receiver, the signature codes of different nano-machines are invoked again to recover the received information. In $[4,5,6]$, the concept of Molecular TimeDivision Multiple-Access (MoTDMA) was proposed to assist multiple nano-machines to transmit information to one common receiver. In MoTDMA, different nanomachines are scheduled to transmit information within different time slots, and hence implement multiple access MC. Moreover, Molecular Division Multiple Access (MDMA) was studied in [7], where a specific type of molecule or a set of molecular types is employed by each individual nano-machine to send its information to a common receiver. Hence, MDMA differentiates different nano-machines via molecular types.

In [8], we have introduced a multiple-access Diffusionbased Molecular Communication (DMC) system based on molecular type hopping and molecular shift-keying modulation, referred to as the MTH-MoSK DMC system. As in MDMA systems, a MTH-MoSK DMC system also exploits multiple types of molecules. However, an MTHMoSK DMC system dose not work on the principle of MDMA systems, in which each transmitter nano-machine uses one exclusive type of molecule. Instead, in MTHMoSK DMC systems, all transmitter nano-machines share all types of molecules. In more detail, in MTH-MoSK DMC systems, each transmitter nano-machine is assigned a unique address code to control the Molecular-Type Hopping (MTH) over, say, $L$ chips (or slots) of one symbol duration, so as to transmit the same symbol $L$ times using $L$ different types of molecules. This processing allows to mitigate Inter-Symbol Interference (ISI) and simultaneously support multiple nano-machines. At the receiver, the information received from different nano-machines is detected by invoking the MTH codes of different nanomachines for molecular type dehopping and with the aid of some other processing.

In multiple access DMC systems, all nano-machines share one DMC channel, resulting in Multiple Access Interference (MAI), which makes reliable detection challenging $[3,9]$. In literature, various detection schemes have been proposed and studied in the context of different multiple-access schemes and channel environments [2, $4,6,7]$. Specifically, the authors of [2] proposed a chip detection scheme, which employs an adaptable threshold derived from the number of molecules received within 
the previous chip. In [4], the detection method presented in [10] was considered for MoTDMA systems. In this detection scheme, the number of received molecules is compared with a variable threshold, which is directly set as the number of molecules of the same type that are received in the previous transmission period. For the MTH-MoSK DMC system proposed in [8], two detection schemes using the principles of majority vote and EqualGain Combining (EGC) were designed and studied. It was shown that the detection performance of both detection schemes is severely affected by MAI.

Therefore, in this paper, we introduce a low-complexity detection scheme which has an MAI mitigation capability for MTH-MoSK DMC systems. Specifically, we investigate and compare two types of low-complexity detection schemes. As the baseline, the first one is the above-mentioned EGC assisted detection [8], which has low complexity but experiences MAI. The second one is also in the principle of EGC but assisted by interference mitigation processing, which is therefore referred to as EGC-IM for convenience of description. Our further discourse will show that the EGC-IM scheme only imposes a slight increase of computation on the EGC scheme, but employs the capability to effectively mitigate MAI. Our simulation results show that MAI dominates the achievable performance, when Signal-to-Noise Ratio (SNR) is relatively high. In this case, the EGC-IM scheme can significantly outperform the conventional EGC scheme.

The contribution of this paper can be briefly summarized as follows:

- An EGC-IM detection scheme is proposed for MTHMoSK DMC systems. The EGC-IM scheme has low complexity, which is similar to the conventional EGC scheme. However, the EGC-IM scheme employs the capability of MAI mitigation.

- The detection performance of an EGC-IM scheme is investigated and compared with that of the conventional EGC scheme, showing that the EGC-IM scheme is a high-efficiency detection scheme for MTH-MoSK DMC to simultaneously support multiple nano-machines.

The remainder of the paper is presented as follows. Section 2 introduces the DMC channel model and the principle of MTH-MoSK DMC. In Section 3, we present the principles of the conventional EGC and the proposed EGC-IM schemes. Performance results are demonstrated in Section 4. Finally, the main conclusions from research are summarized in Section 5.

\section{SYSTEM MODEL}

\subsection{Transmitted signal}

The system diagram for the MTH-MoSK system is shown in Fig. 1. We assume that the MTH-MoSK DMC system employs $M$ types of information molecules for implement-

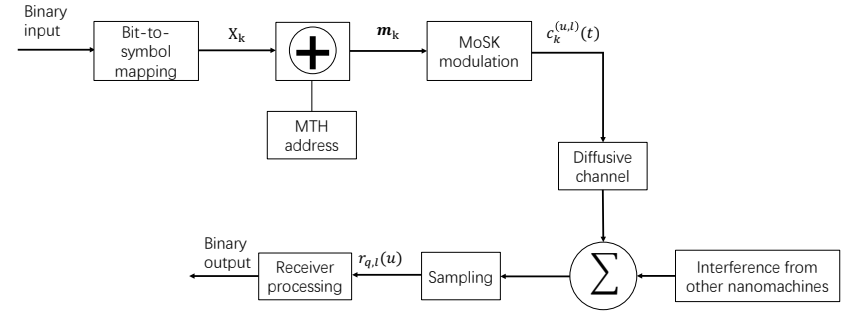

Fig. 1 - System diagram showing the components of MTH-MoSK DMC systems.

ing joint MTH-assisted multiple access and MoSK modulation, where each transmitted symbol conveys $\log _{2} M$ bits. In an MTH-MoSK DMC system, the symbol duration of $T_{s}$ seconds is divided into $L$ chips of each lasting $T_{h}=T_{s} / L$ seconds. We assume that the system supports $K \leq M$ nano-machines and they have a similar distance $d$ from a common Access Point (AP), which may be connected with other communication networks.

As illustrated in Fig. 1, $\log _{2} M$ bits of binary information are mapped to a $M$-ary symbol $X_{k} \in$ $\{0,1, \ldots, M-1\}$ to be transmitted by the $k$ th nanomachine. Using the unique MTH address code assigned to the $k$ nano-machine, which is expressed as $\boldsymbol{a}_{k}=$ $\left[a_{k}^{(0)}, a_{k}^{(1)}, \ldots, a_{k}^{(L-1)}\right], a_{k}^{(i)} \in[0, M-1]$, the symbol $X_{k}$ is signed to give

$$
\begin{aligned}
\boldsymbol{m}_{k}= & {\left[m_{k}^{(0)}, m_{k}^{(1)}, \ldots, m_{k}^{(L-1)}\right] } \\
= & X_{k} \cdot \mathbf{1}_{(1 \times L)} \oplus \boldsymbol{a}_{k} \\
= & {\left[X_{k} \oplus a_{k}^{(0)}, X_{k} \oplus a_{k}^{(1)}, \ldots, X_{k} \oplus a_{k}^{(L-1)}\right], } \\
& k=1,2, \ldots, K
\end{aligned}
$$

where $\oplus$ presents the addition operation in the Galois field GF $(M)$ [11] and $\mathbf{1}_{(1 \times L)}$ is an all-one-element row vector of length $L$. Therefore, the elements of $m_{k}$ take values in $\{0,1, \ldots, M-1\}$. After the MTH processing, $M$-ary MoSK modulation is implemented, as shown in Fig. 1, and the corresponding types of molecules are successively emitted into the DMC environment from $l=0$ to $l=L-1$. To explain the principles in more detail, we consider an MTH-MoSK DMC system supporting $K=2$ nano-machines, whose MTH address codes are $\boldsymbol{a}_{1}=[6,2,5,7,3,4]$ and $\boldsymbol{a}_{2}=[0,1,7,3,6,2]$, respectively. The symbols transmitted by the two nano-machines are $X_{1}=7$ and $X_{2}=2$, respectively. Then, after the addition operations in the Galois field, we obtain $m_{1}=$ $[1,5,2,0,4,3]$ and $\boldsymbol{m}_{2}=[2,3,5,1,4,0]$, which determine which type of molecule is emitted within a chip of a symbol duration.

Specifically, if within the $l$ th chip of the $u$ th symbol duration, the type- $q$ molecules is emitted by the $k$ th nanomachine to transmit information, according to Fick's law $[12,13]$, the concentration of the $q$ th type of information molecules sampled at time $t \geq(u L+l) T_{h}$ can be expressed as 


$$
\begin{aligned}
c_{k, q}^{(u, l)}(t) & =\frac{A}{\left[4 \pi D\left(t-u T_{s}-l T_{h}\right)\right]^{\frac{3}{2}}} \\
& \times \exp \left(\frac{-d^{2}}{4 D\left(t-u T_{s}-l T_{h}\right)}\right),
\end{aligned}
$$

where $D$ is the diffusion coefficient of information molecules, $A$ is the number of molecules emitted per molecular pulse and $d$ is the distance between a transmit nano-machine and the AP.

Due to the free diffusion of molecules, there are possibly three kinds of interference encountered in MTH-MoSK systems. The first kind originates from the same type of molecules emitted repetitively, which generates InterSymbol Interference (ISI). The second one is the noise resulted from molecular Brownian motion. Finally, if several nano-machines transmit signals depending on the same type of molecules within one given chip, the signals transmitted by different nano-machines interfere with each other. Moreover, because the large delay-spread of molecular communication channels, the same type of molecules emitted by different nano-machines in previous chips may also interfere with the signals transmitted within the current chip. These kinds of interference caused by multiple nano-machines are all classified as Multiple-Access Interference (MAI). Hence, in order to recover the information transmitted by different nanomachines, the design of an efficient detector in MTHMoSK DMC systems is critical.

\subsection{Received observations}

For simplicity, the receiver at the AP is assumed to be constructed by a spherical sensing space with the radius of $\rho$, which is able to ideally measure the concentrations of the different types of molecules presenting in the space. We assume that $\rho$ is small enough with respect to the communication distance, so that near-uniform concentrations present in the sensing space. In other words, the sensing space is idealized as a point space. Based on these assumptions, the received peak concentration within each chip is expected to occur at the time $t_{d}=\frac{d^{2}}{6 D}$ from the start of each chip [13], which is usually used as the observation for information detection. Following this convention, the observation obtained during the lth chip of the $u$ th symbol duration is given by

$$
\begin{gathered}
r_{q, l}(u)=r_{q}\left(t=u T_{s}+l T_{h}+t_{d}\right), l=0,1, \ldots, L-1 ; \\
u=0,1, \ldots ; q=0,1, \ldots, M-1
\end{gathered}
$$

To be more specific, when taking account the effect from Brownian motion noise, the ISI from the desired nanomachine and the MAI generated by other nano-machines, the received observation $r_{q, l}(u)$ can be expressed as

$$
\begin{aligned}
r_{q, l}(u) & =\sum_{k=1}^{K} \sum_{i=0}^{\min \{I, u L+l\}} \ell_{k, q}^{u L+l-i} c_{k, q}\left(i T_{h}+t_{d}\right) \\
& +n_{q}\left(u T_{s}+l T_{h}+t_{d}\right) \\
& =\sum_{k=1}^{K} \sum_{i=0}^{\min \{I, u L+l\}} \ell_{k, q}^{u L+l-i} c_{k, q}(i)+n_{q, l}(u)
\end{aligned}
$$

where $I$ is the length of ISI, $\ell_{k, q}^{i}$ makes a logic decision, which is ' 1 ', when the $k$ th nano-machine uses the $q$ th type of molecule to transmit its information within the $i$ th chip, it is '0', otherwise. $c_{k, q}(i)$ is the expected concentration of the $q$ th type of molecule at $t=i T_{h}+t_{d}$, obtained from the Fick's law of (2), after $A$ molecules of $q$ th type was emitted at $t=0$. Note that, $c_{k, q}(0)$ corresponds to the peak concentration of the $q$ th type of molecule in the current chip, while for $i>0$, it contributes interference. Finally, $n_{q, l}$ in (4) is the Brownian motion noise imposed on the $l$ th chip of the $u$ th symbol duration, but relied on the transmission of the $q$ th type of molecules. It is shown that $n_{q, l}$ can be approximated as the Gaussian noise with zero mean and a variance of [14]

$$
\Delta_{q, l}^{2}(u)=\frac{1}{V} \sum_{k=1}^{K} \sum_{i=0}^{\min \{I, u L+l\}} \ell_{k, q}^{u L+l-i} c_{k, q}(i)
$$

expressed as $n_{q, l}(u) \sim \mathcal{N}\left(0, \Delta^{2}{ }_{, l}(u)\right)$. In (5), $V$ represents the volume of the detection sphere of the receiver. Explicitly, the variance of noise is dependent on the transmitted signals, where signal power results in higher noise power.

\section{EQUAL-GAIN COMBINING WITH INTER- FERENCE MITIGATION}

The proposed EGC-IM scheme is an enhanced single-user detection scheme. In comparison with the conventional EGC scheme, EGC-IM requires a slightly increased computation. However, as the results in Section 4 illustrate, it has the potential to mitigate the MAI and ISI existing in our MTH-MoSK system and hence, achieves performance improvement when compared with the conventional EGC scheme.

According to (3), within one symbol duration, we can obtain in total $M \times L$ observations, consisting of the signals transmitted by $K$ nano-machines during the current symbol duration, the interference from previous symbol durations and background noise. Let us collect the $M \times L$ observations obtained during the $u$ th symbol duration to form an observation matrix $R_{u}$, whose $M$ rows represent $M$ molecular types and $L$ columns denote the $L$ chips of the $u$ th symbol duration. Furthermore, the $(q, l)$ th element of $\boldsymbol{R}_{u}$ is given by $r_{q, l}(u)$.

The principle of the conventional EGC can be found in many references [15]. With the aid of Fig. 2, the principle 


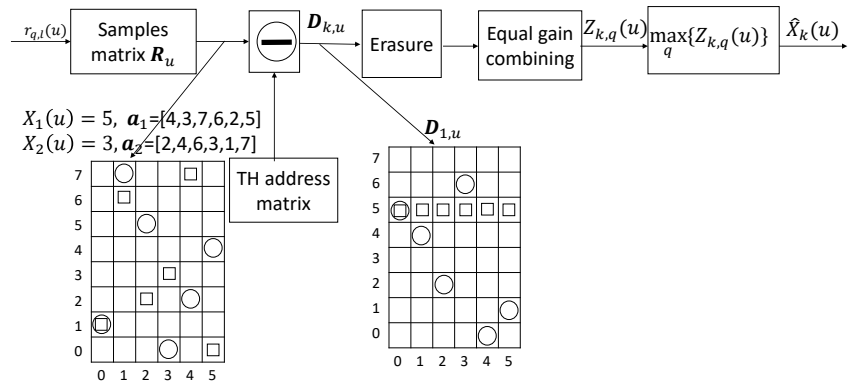

Fig. 2 - Schematic block diagram showing the EGC-IM assisted detection.

of EGC-IM can be described as follows. Let us assume that there are $K=2$ nano-machines and their MTH addresses are $a_{1}=[4,3,7,6,2,5]$ and $a_{2}=[2,4,6,3,1,7]$, respectively; The symbols sent by these two nano-machines are $X_{1}(u)=5$ and $X_{2}(u)=3$. Then, the observation matrix $\boldsymbol{R}_{\boldsymbol{u}}$ has the form shown in Fig. 2, where the square and circle elements are respectively activated by the irst and second nano-machines. From previous description we can know that these marked elements consist of signal, interference and noise, while the other empty elements contain only noise and interference.

Having obtained the observation matrix $\boldsymbol{R}_{u}$, next, we can invoke the MTH address code $\boldsymbol{a}_{k}$ of the desired nanomachine $k$ (to be detected) to de-hop the observation matrix $\boldsymbol{R}_{u}$, generating the detection matrix $D_{k, u}$ for nanomachine $k$. To be more speciic, the de-hopping operation shifts the $(q, l)$ th element $r_{q, l}(u)$ in $\boldsymbol{R}_{u}$ to the new location $\left(q \ominus a_{k}(l), l\right)$ in $\boldsymbol{D}_{k, u}$, which can be described as

$$
\begin{aligned}
d_{\left(q \ominus a_{k}(l), l\right)}^{(k)}(u) & =r_{q, l}(u), q=0,1, \ldots, M-1 ; \\
l & =0,1, \ldots, L-1 ; k=1,2, \ldots, K
\end{aligned}
$$

Speciicall y for the example shown in Fig. 2, after applying the MTH address $\boldsymbol{a}_{1}=(4,3,7,6,2,5)$ of the irst nanomachine to de-hop $R_{u}$, the detection matrix $D_{1, u}$ is obtained, as shown in Fig. 2. Explicitly, all the elements activated by the irst nano-machine are shifted to the 5 th row, whose index explains that the $M$-ary symbol transmitted by the irst nano-machine is $X_{1}(u)=5$. By contrast, the elements activated by the second nano-machine scatter randomly in different rows.

It can be reasoned that the elements activated by the interfering nano-machines should be expected to have higher energy than the elements containing only interference plus noise. Hence, in the EGC-IM assisted detection, we may make use of this property to mitigate MAI. Speciicall y, to detect a nano-machine's information, we irst identify the $J(J<L)$ largest elements in each row of $D_{k, u}$ and substitute them with zeros. Following the above analysis, this operation will most probably remove the elements activated by the interfering nano-machines, or the elements experiencing high interference due to ISI and MAI. This can be understood with the aid of the example shown in Fig. 2. For principle, we assume that there is no ISI and one maximum element of each row is removed. Then, we can know that all the interfering elements in rows $0,1,2,4$ and 6 will be removed. At the same time, the maximum of Row 5 is removed, which will reduce the output of inal combining, as shown below. However, Row 5 still has 5 elements containing the desired signal and the symbol of nano-machine 1 can be detected free from interference.

Let the detection matrix of nano-machine $k$ after the IM operations be expressed as $D_{k, u}^{\prime}$ with its elements as $\bar{d}_{(q, l)}^{(k)}(u)$. Then, based on $\boldsymbol{D}_{k, u}^{\prime}$, EGC is implemented to form $M$ decision variables as

$$
\begin{aligned}
D_{q}^{(k)}(u)=\sum_{l=0}^{L-1} \bar{d}_{(q, l)}^{(k)}(u), q & =0,1, \ldots, M-1 ; \\
k & =1,2, \ldots, K
\end{aligned}
$$

for each of $u=0,1, \ldots$ The last step of the EGC-IM assisted detection is to select the largest of the $M$ decision variables $\left\{D_{0}^{(k)}(u), D_{1}^{(k)}(u), \ldots, D_{M-1}^{(k)}(u)\right\}$ provided by (7), whose index for $q$ represents the detected $M$-ary symbol transmitted by the desired nano-machine $k$. This is expressed as

$$
\hat{X}_{k}(u)=\arg \max _{q}\left\{D_{q}^{(k)}(u)\right\}, k=1,2, \ldots, K
$$

where $D_{q}^{(k)}(u) \in\left\{D_{0}^{(k)}(u), D_{1}^{(k)}(u), \ldots, D_{M-1}^{(k)}(u)\right\}$. Note that, as mentioned above, the erasure operation can mitigate MAI/ISI, while also reduce the output value of the desired decision variable, yielding a trade-off between the value of $J$ and the achievable performance. Hence, for a given Signal-to-Noise (SNR), a given number $K$ of nanomachines and a given number $L$ of chips per symbol duration, there exists an optimum value for $J$, which results in the best performance, as to be demonstrated in Section 4 .

\section{PERFORMANCE RESULT}

In this section, the error performance of the MTH-MoSK DMC systems with EGC-IM is demonstrated and compared with that of the MTH-MoSK DMC systems with the conventional EGC. In following igur es, SNR is the ratio between the power of one pulse of molecules released for transmitting one bit information and noise power, given by

$$
\mathrm{SNR}=\frac{c_{b(o)}^{2}}{c_{b(o)} / V}=V c_{b(o)}
$$

where $c_{b(o)}$ is the peak concentration and $V$ is the volume of the receiver detection space.

From (9) we can know that SNR is linearly proportional to the number of molecules $A$ emitted per pulse, as $c_{b(o)}$ is a linear function of $A$, as shown in (2). However, using SNR instead of directly the number of molecules $A$ has the advantage that it can take the volume of detection space (or the capability of receiver) into account. This is because 
in practical communication systems (DMC or other digital communication systems), it is the SNR that determines the achievable performance. No matter how high is the transmit power (i.e., the number of molecules emitted per pulse in DMC), the communication systems' performance will be poor, if the SNR generated by the receiver is low. To set the parameters in our simulations, when given an SNR and a volume $V$ of detection space, $c_{b(o)}$ is calculated by (9). Then, when given a transmission distance $r_{k}$ between nano-machines and AP, the number of molecules $A_{b}$ emitted to transmit one bit can be obtained with the aid of (2). Since an MTH-MoSK DMC system employs the $M$-ary MoSK to transmit $\log _{2} M$ bits per symbol, a nanomachine can emit in total $A_{s}=\log _{2} M \times A_{b}$ molecules for transmission of one symbol. However, as one symbol is transmitted by $L$ pulses in $L$ chips, the number of molecules $A_{h}$ emitted per chip is $A_{h}=A_{s} / L$.

Additionally, there are some parameters fixed in our simulations, these include the similar distance of $d=250 \mathrm{~nm}$ from each nano-transmitter to AP, $D=2.2 \times 10^{-9} \mathrm{~m}^{2} / \mathrm{s}$ and $V=\frac{4}{3} \pi \rho^{3}$ with $\rho=20 \mathrm{~nm}$. Moreover, we set the bit duration as $T_{b}=6 \times 10^{-5} \mathrm{~s}$, giving the symbol duration of $T_{s}=\log _{2} M \times\left(6 \times 10^{-5}\right)(s)$. Hence, when there are $L$ chips per symbol, the chip duration is given by $T_{h}=T_{s} / L=\log _{2} M \times\left(6 \times 10^{-5}\right) / L(s)$. Finally, the length $I$ of ISI is set as the value of

$$
I \triangleq \arg _{i}\left\{\frac{c_{h(i)}}{c_{h(o)}} \leq 0.1 \%\right\}
$$

which means that the interference $30 d B$ below the signal power is ignored.

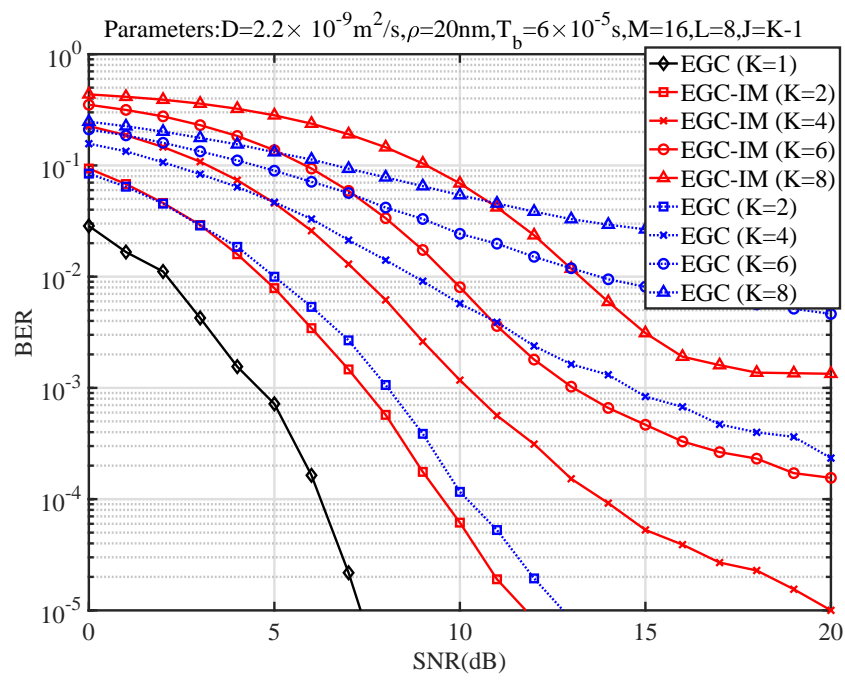

Fig. 3 - Comparison of BER versus SNR performance of the MTH-MoSK DMC systems with EGC-IM and with conventional EGC, when different numbers of nano-machines are supported.

First, in Fig. 3, we demonstrate and compare the BER versus SNR performance of the MTH-MoSK DMC systems employing the proposed EGC-IM and the conventional EGC, respectively, when $K=1,2,4,6$ or 8 nano-machines are supported. When EGC-IM is employed, we assume
$J=K-1$ elements are removed from each row of the detection matrix $D_{k, u}$. According to the results shown in Fig. 3, we have the following observations. First, the BER performance of the MTH-MoSK DMC systems employing either detection schemes degrades with the increase of the number of nano-machines, as the result of MAI. When $K \geq 2$, the EGC-IM scheme outperforms the conventional EGC scheme, provided that SNR is sufficiently high. This observation becomes clearer when $K$ is larger and SNR is higher. However, if SNR is low, the EGC-IM may be outperformed by the conventional EGC scheme. The reason behind this is that when SNR is low, system performance is dominated by background noise. In this case, combining more samples encourages smooth noise and hence improves BER performance. By contrast, when SNR is relatively high, system performance is dominated by MAI. Hence, the proposed EGC-IM scheme with the capability of MAI mitigation outperforms the conventional EGC detection.

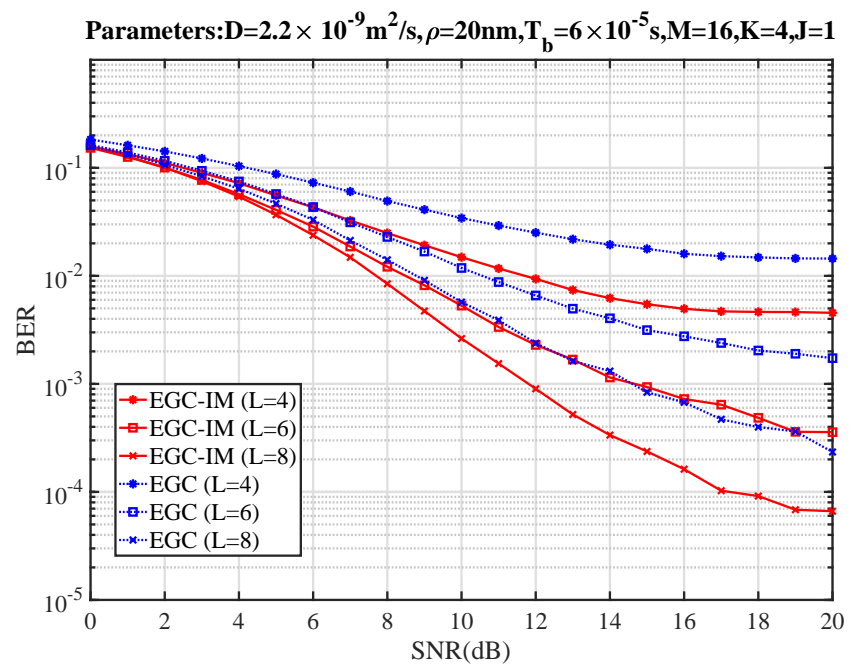

Fig. 4 - BER versus SNR performance of the MTH-MoSK DMC systems with the conventional EGC and the proposed EGC-IM schemes, when one symbol is transmitted using $L=4,6$ or 8 chips.

Second, we investigate the effect of $L$ on the performance of the considered detection schemes in Fig. 4. Given $K=$ 4 nano-machines supported and $J=1$ entry per row removed from the detection matrix, the proposed EGC-IM always outperforms the conventional EGC within the considered SNR region for all the $L$ values. Furthermore, the results show that for both the EGC-IM and EGC, the BER performance improves, as the value of $L$ increases. This is the result that MAI decreases as $L$ increases.

Fig. 5 demonstrates the impact of the number of molecular types on the BER performance of the MTH-MoSK DMC systems supporting $K=4$ nano-machines. As shown in the figure, when an EGC-IM scheme is employed, $J=1$ entry is removed from each row of the detection matrix. The results in Fig. 5 demonstrate that as more types of molecules are employed, the BER performance of MTHMoSK DMC systems improves, in addition to the increased 


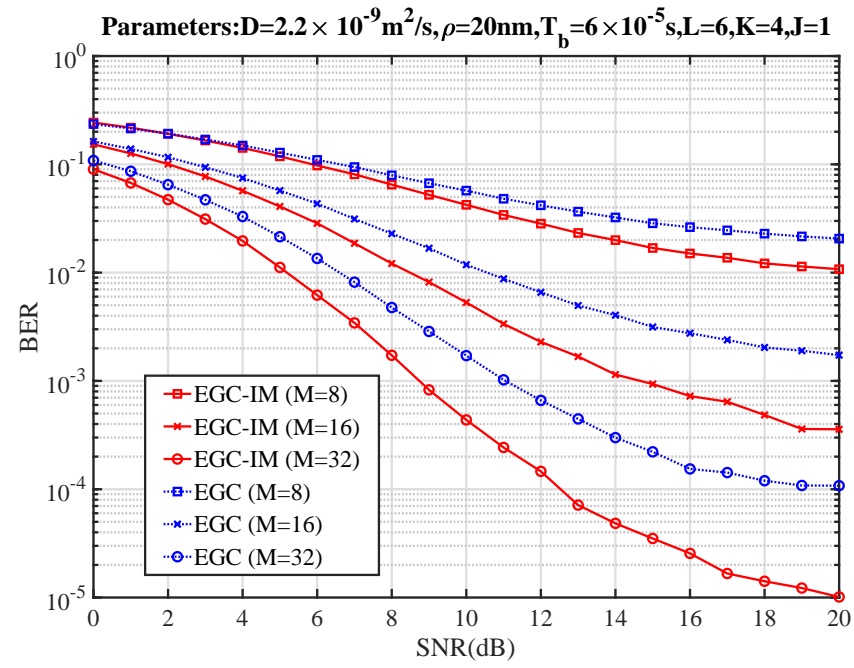

Fig. 5 - BER versus SNR performance of the MTH-MoSK DMC systems with the conventional EGC and proposed EGC-IM, when the number of molecular types is $M=8,16$ or 32 .

data rate ${ }^{1}$. The reason behind this is that for a given value of $K$, MAI reduces as $M$ increases. This is because the MAI entries are more sparsely distributed in the detection matrix, as implied in Fig. 2, when $M$ becomes larger. Again, as shown by Fig. 5, the EGC-IM detection scheme outperforms the conventional EGC scheme and furthermore, the performance gain at a given SNR increases with the increase of $M$.

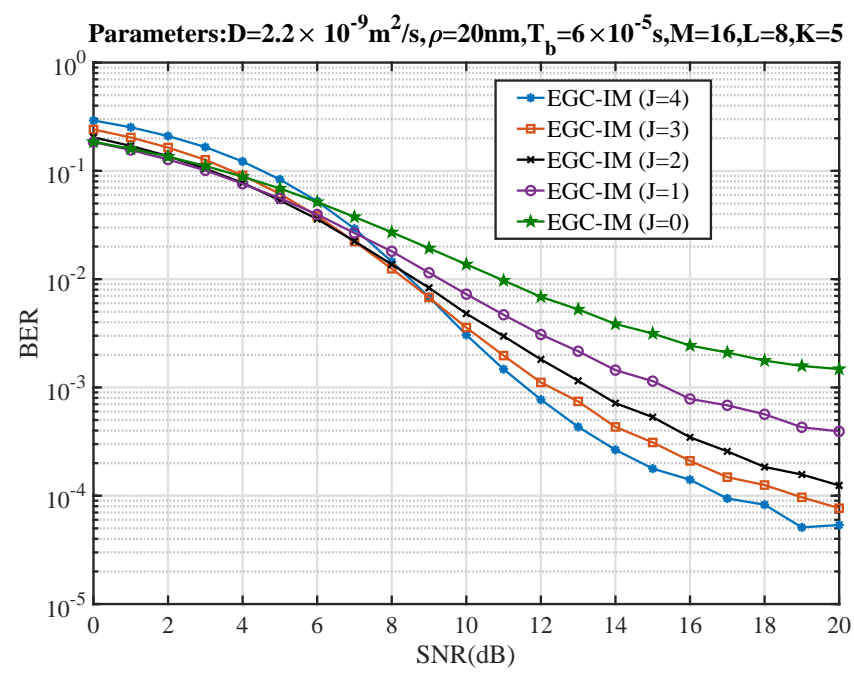

Fig. 6 - BER versus SNR performance of the MTH-MoSK DMC systems with the EGC-IM, when different number of elements per row are removed from the detection matrix.

Finally, Fig. 6 demonstrates the effect of the number of elements removed from each of the rows in the detection matrix $\boldsymbol{D}_{k, u}$ on the BER performance of the MTH-MoSK DMC systems with the proposed EGC-IM. In the considered MTH-MoSK DMC systems, we assume that there are $K=5$ nano-machines supported. Note that, when $J=0$, the EGC-IM is reduced to the conventional EGC. The re-

\footnotetext{
${ }^{1}$ When $M=8,16$ and 32 , the data rates are 3,4 , and 5 bits per symbol, respectively.
}

sults of Fig. 6 show that the BER drops, as the value of $J$ increases, provided that the SNR is sufficiently high. This observation implies that MAI can be efficiently mitigated by deleting an appropriate number of entries from the detection matrix. However, at the low SNR region, the EGCIM with more elements removed from each row of the detection matrix might not result in BER performance improvement. In other words, for a given SNR and a given value of $K$, there is an optimum value of $J$ to attain the best possible error performance.

\section{CONCLUSIONS}

In this paper a novel EGC-IM detection scheme has been proposed for MTH-MoSK DMC systems. We have investigated the BER achievable by the EGC-IM and compared it with that of the conventional EGC-aided detection scheme. The results show that in the MTH-MoSK DMC systems supporting multiple nano-machines, the EGC-IM scheme is capable of achieving much better BER performance than the conventional EGC scheme within the practical SNR region resulting in a BER below $10^{-2}$. Therefore, the proposed EGC-IM scheme is efficient for MAI mitigation. Furthermore, the proposed EGC-IM has low complexity. In comparison with the conventional EGC scheme, the extra operations required by the EGC-IM scheme are only identifying some of the largest elements in each row and deleting them. In addition, for given values of $M, L, K$ and SNR, there is an optimum number of deleted elements from each row to achieve the best BER performance. Our future research will consider the effect of the different distances from distributed nano-machines as well as the design of the extended IM schemes that are efficient for operation in this communication scenario.

\section{REFERENCES}

[1] G. K. Walia, D. K. K. Randhawa, and K. S. Malhi. "A brief survey on molecular communications in nanonetworks". In: 2016 International Conference on Computational Techniques in Information and Communication Technologies (ICCTICT). Mar. 2016, pp. 343-348.

[2] Y. Zamiri-Jafarian, S. Gazor, and H. Zamiri-Jafarian. "Molecular code division multiple access in nano communication systems". In: 2016 IEEE Wireless Communications and Networking Conference. Apr. 2016, pp. 1-6.

[3] L. Wang and A. W. Eckford. "Nonnegative code division multiple access techniques in molecular communication". In: 2017 15th Canadian Workshop on Information Theory (CWIT). June 2017, pp. 1-5.

[4] S. Korte, M. Damrath, M. Damrath, and P. A. Hoeher. "Multiple Channel Access Techniques for DiffusionBased Molecular Communications". In: SCC 2017; 11th International ITG Conference on Systems, Communications and Coding. Feb. 2017, pp. 1-6. 
[5] H. Tezcan, S. Oktug, and F. N. Kok. "Neural delay lines for TDMA based molecular communication in neural networks". In: 2012 IEEE International Conference on Communications (ICC). June 2012, pp. 6209-6213.

[6] R. Yu, M. S. Leeson, and M. D. Higgins. "Multipleaccess scheme optimisation for artificial neuronal networks". In: 2014 9th International Symposium on Communication Systems, Networks Digital Sign (CSNDSP). July 2014, pp. 428-433.

[7] G. Aminian, M. Farahnak-Ghazani, M. Mirmohseni, M. Nasiri-Kenari, and F. Fekri. "On the Capacity of Point-to-Point and Multiple-Access Molecular Communications With Ligand-Receptors". In: IEEE Transactions on Molecular, Biological and MultiScale Communications 1.4 (Dec. 2015), pp. 331346.

[8] Weidong Gao, Terrence Mak, and Lie-Liang Yang. "Molecular Type Spread Molecular Shift Keying for Multiple-Access Diffusive Molecular Communications". In: IEEE Transactions on Molecular, Biological and Multi-Scale Communications 7.1 (2021), pp. 51-63.

[9] C. Jiang, Y. Chen, and K. J. R. Liu. "Inter-user interference in molecular communication networks". In: 2014 IEEE International Conference on Acoustics, Speech and Signal Processing (ICASSP). May 2014, pp. 5725-5729.

[10] M. Damrath and P. A. Hoeher. "Low-Complexity Adaptive Threshold Detection for Molecular Communication". In: IEEE Transactions on NanoBioscience 15.3 (Apr. 2016), pp. 200-208.

[11] D. J. Goodman, P. S. Henry, and V. K. Prabhu. "Frequency-hopped multilevel FSK for mobile Radio". In: The Bell System Technical Journal 59.7 (Sept. 1980), pp. 1257-1275.

[12] L. Shi and L. Yang. "Diffusion-based molecular communications: Inter-symbol interference cancellation and system performance". In: 2016 IEEE/CIC International Conference on Communications in China (ICCC). July 2016, pp. 1-6.

[13] I. Llatser, A. Cabellos-Aparicio, M. Pierobon, and E. Alarcon. "Detection Techniques for Diffusionbased Molecular Communication". In: IEEE Journal on Selected Areas in Communications 31.12 (Dec. 2013), pp. 726-734.

[14] L. Meng, P. Yeh, K. Chen, and I. F. Akyildiz. "On Receiver Design for Diffusion-Based Molecular Communication". In: IEEE Transactions on Signal Processing 62.22 (Nov. 2014), pp. 6032-6044.

[15] Lie-Liang Yang. Multicarrier communications. John Wiley \& Sons, 2009.

\section{AUTHORS}

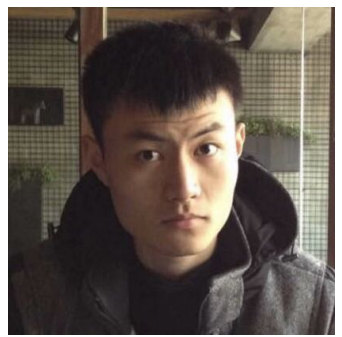

Weidong Gao received his B.Eng degree in electronics and information from the Soochow University, Suzhou, China, in 2016 and his M.Sc degree in electronic engineering from the University of Southampton, Southampton, U.K., in 2017, where he is currently pursuing a Ph.D degree. His research interest is in molecular communications, in particular, in diffusive molecular communications.

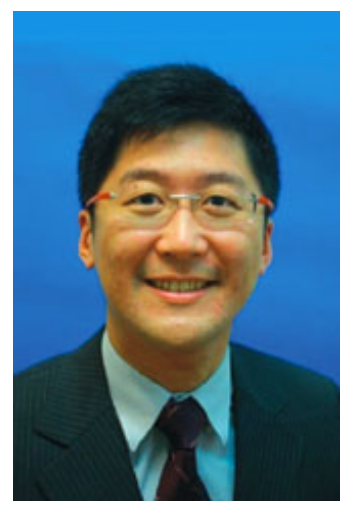

Terrence Mak is currently an Associate Professor with the Department of Electronics and Computer Science, University of Southampton, Southampton, U.K. He graduated at Imperial College London for his $\mathrm{PhD}$ and is a Visiting Scientist with Massachusetts Institute of Technology, Cambridge, MA, USA, in 2010. He has been granted two U.S. and one U.K. patent for his engineering designs, i.e., US 16/685 090 and US 13/638 330. He has published more than 150 papers in both conferences and journals and jointly published four books. His research areas include computer architecture design, optimization and adaptation for VLSI systems, network-on-chip, 3-D Integrated Circuits (ICs), and, lately, wireless-on-chip. Prof. Mak received the IET Premium Yearly Best Paper Award for Computer \& Digital Techniques in 2013, and his newly published journal based on 3D-IC received the prestigious 2015 IET Computers \& Digital Techniques Premium Award. Throughout a spectrum of publications, he has been awarded six best paper awards, and one nominated, from prestigious conferences, at EMBS 2005, DATE 2011, VLSI-SoC 2014, PDP 2015, EUC 2016, DATE 2018 (nominated), and ISPLED 2019. His publication for IEEE Transactions was selected as a "Top 25 Downloaded Manuscript" in 2015. 


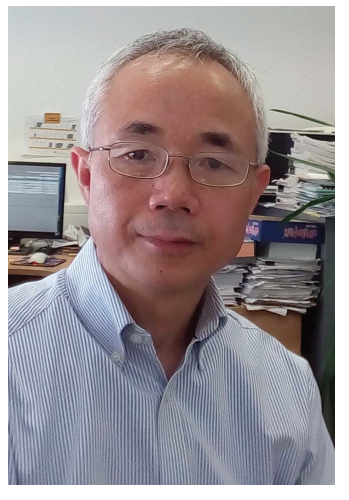

Lie-Liang Yang received his BEng degree in communications engineering from Shanghai TieDao University, Shanghai, China in 1988, and his MEng and $\mathrm{PhD}$ degrees in communications and electronics from Northern (Beijing) Jiaotong University, Beijing, China in 1991 and 1997, respectively. From June 1997 to December 1997 he was a visiting scientist to the Institute of Radio Engineering and Electronics, Academy of Sciences of the Czech Republic. Since December 1997, he has been with the University of Southampton, United Kingdom, where he is the professor of Wireless Communications in the School of Electronics and Computer Science. He has research interest in wireless communications, wireless networks and signal processing for wireless communications, as well as molecular communications and nano-networks. He has published over 400 research papers in journals and conference proceedings, authored/co-authored three books and also published several book chapters. The details about his research publications can be found at https://www.ecs.soton.ac.uk/people/llyang. He is a fellow of both the IEEE and the IET, and was a distinguished lecturer of the IEEE VTS. He served as an associate editor to the IEEE Trans. on Vehicular Technology and Journal of Communications and Networks (JCN), and is currently an associate editor to the IEEE Access and a subject editor to the Electronics Letters. 